\title{
Meeting Physician Compliance Recommendations in the Management of Opioids in Chronic Pain: The Chronic Pain Management Registry (CPMR)
}

Paul C Langley, PhD, Adjunct Professor

College of Pharmacy University of Minnesota

\begin{abstract}
In a recent commentary in INNOVATIONS in Pharmacy, details were given on a recently released Chronic Pain Management Registry (CPMR). The CPMR was designed to provide a tracking and audit framework for evaluating claims made for therapy interventions in chronic pain management. At the same time, the CPMR was seen as a key element in monitoring physician and practice compliance with requirements for the prescribing of opioids and other scheduled substances. The purpose of the present commentary is to expand upon the role of the CPMR in the management of opioids in detailing the concordance of the CPMR data collection requirements with the latest recommendations of the American Society of Interventional Pain Physicians (ASIPP) for responsible, safe and effective opioid prescribing in chronic non-cancer pain. Given ongoing concerns with opioid misuse and abuse, the opioid epidemic, physician practices are at risk for what may be judged as poor therapy decisions in evaluating medical necessity and a failure to monitor effectively response to therapy. Adoption of a platform such as the CPMR may, through providing a comprehensive evidence base and tracking capability, support more effective prescribing decisions and adherence to therapy. At the same time, the ability to justify decisions through a CPMR documentation audit may not only alleviate physician concerns if their decisions are challenged but also lead to improved outcomes in the treatment of chronic pain.
\end{abstract}

Keywords: Chronic Pain Management Registry, CPMR, ASIPP Guidelines, physician compliance, opioid evaluation, pain management

\section{Introduction}

Compliance with guidelines for opioid and other controlled substance prescribing decision in the management of chronic non-cancer pain are of continuing concern for individual physicians, physician practices and health care systems. A particular focus of this concern is with providing a readily accessible audit trail to justify prescribing decisions involving opioids. The Medical Board of California, to give one example, has since the mid-1990s attempted to provide guidance to physicians prescribing controlled substances for pain ${ }^{1}$. The most recent edition of these guidelines points to the requirement under California law that 'the people of California have access to appropriate, safe and effective pain management' and that, with the complexity inherent in treating chronic pain "long-term opioid therapy should only be conducted in practice settings where careful evaluation, regular follow-up, and close supervision are ensured'. The California guidelines point out that 'Medicine is practiced one patient at a time and each patient has individual needs and vulnerabilities. Physicians are encouraged to document their rationale for each prescribing decision. Physicians should understand that if one is ever the subject of a quality of care

Corresponding author: Paul C Langley, PhD

Adjunct Professor

College of Pharmacy University of Minnesota

Director, Maimon Research LLC

5061 North Apache Hills Trail, Tucson, AZ 85750

Email: langley@maimonresearch.com complaint, peer expert review will be sought by the Board. The expert reviewer must consider the totality of circumstances surrounding the physician's prescribing practice'. California is not, of course, alone among the states in expressing these concerns with the need to justify prescribing decisions. Added weight is given by the role of the federal government through agencies such as the Centers for Disease Control and the Substance Abuse and Mental Health Services Administration and penalties under Title 21 United States (USC) Controlled Substances Act.

Documentation to support prescribing decisions is a key element in the treatment of chronic pain. This is important, not only in responding to quality of care complaints and investigations in opioid prescribing by physicians and physician practices, but also in assessing, not just the logic behind an initial prescribing decision but in tracking or monitoring the patient over the course of their therapy. Apart from closer management of patients to assess potential medication abuse, the audit trail is critical in evaluating the credibility of claims made by manufacturers for competing products and procedures in terms of pain level and functional status. Unfortunately, the evidence base for medium and long-term chronic pain interventions is limited ${ }^{2}$. The more robust evidence is for short-term clinical trials, which all too often lack external validity in translating their claims to target population. Beyond 12 weeks, the typical pain study protocol length, the evidence for response and risk/benefit calculation is sparse. This places a premium on the ability of physicians and pain practices to generate evidence to support therapy decisions and intervene to modify or switch therapy. 
Understandably, manufacturers are reluctant to support longer term assessments once a product or procedure has received marketing authorization; the responsibility, therefore, falls on health systems as well as individuals to generate the evidence.

The absence of medium and long term clinical trial (as well a dearth of observational study data) puts the onus on treating physicians not only to justify an initial opioid prescribing decision but to justify continuing to prescribe opioids. This puts the emphasis on response to therapy. Ideally targets for therapy should have been agreed with the patient as part of a pain management plan (e.g., returning to work). Tracking the patient over time and monitoring the response to treatment is, presumably, a key component. This response, in terms of the impact of therapy on pain level by pain location(s) and functional status should be tracked against baseline presentation with a determination at each visit, together with the patient's perception of response. Monitoring both clinical response and the patient's subjective assessment is important, not only because limited evidence would suggest that the two might diverge but that a patient's subjective assessment may impact aberrant medication behavior ${ }^{3}$. In addition, as comorbidities such as depression and anxiety may also impact medication behavior, these should also be monitored as part of a comprehensive pain management plan.

At the same time, access to documentation to support prescribing can also support appeals where opioid prescribing has been denied. This provides additional flexibility in challenging third-party decisions when the treating physician has determined and can justify a particular therapy choice together with a demonstrated ability to monitor closely the patient's response to therapy as further support for the decision.

The purpose of this commentary is to complement a recent publication in INNOVATIONS in Pharmacy that detailed the development of a cloud-based Chronic Pain Management Registry (CPMR) ${ }^{4}$. This registry has as its principal role the monitoring and tracking of therapy outcomes in chronic pain. At the same time, it has been designed to support the prescribing information and documentation needs of physicians and physician practices in meeting compliance standards in opioid utilization. In the present commentary the evidence base generated by the CPMR is considered in terms of evidence recommendations for compliance proposed by the American Society of Interventional Pain Physicians (ASIPP) in their latest guidelines for responsible, safe and effective opioid prescribing in non-cancer pain ${ }^{5}$.

A caveat, however, is in order: the CPMR is designed to do more than provide an evidence base to ensure physician compliance with opioid management recommendations and requirements. The ability to track outcomes is a key input to establishing the credibility of claims for competing pain management interventions. These comparisons could be between the various opioid formulations, between the various interventional procedures with their claims for 'instant' pain relief and between pharmacological and non-pharmacological interventions in the medium to long term. Adoption of the CPMR by primary care physicians as well as by specialist pain clinics allows for claims to be assessed and replicated in different treating environments across target patient groups.

\section{The Chronic Pain Management Registry (CPMR)}

As detailed in the companion paper, the CPMR captures data on-line from both patients and physician practices ${ }^{2}$. Patients complete an on-line report prior to each physician visit with a summary submitted immediately to the physician practice. Subsequent to the visit, the patient completes an on-line satisfaction with care report with, for the first visit, a series of questions to determine the extent to which a pain management plan was discussed and implemented. The initial patient report captures: health status, pain experience, chronic pain status, pain location, pain and functional status by pain location, opioid use, attitude to medications, risk assessment for the likelihood of aberrant opioid use and comorbidity status (specifically fatigue, depression, anxiety, sleep experience and constipation). Subsequent to the initial visit, the follow-up patient reports focus on recording pain status and functionality by pain location, aberrant medication use, symptom and comorbidity status and the patients belief in the efficacy of the treatment using the Patient's Global Impression of Change (PGIC) scale ${ }^{6}$. Follow-up summary reports are sent to the physician practice detailing change in pain and functional status by pain location over baseline, reported medication aberrant behavior, changes in symptom and comorbidity status and perceived response to therapy since therapy initiation.

Following each visit, the physician practice also completes an on-line report on the patient. These data are integrated with the patient's reports to create the registry. The data elements captured include: pain evaluation, medication utilization, adverse events, ICD-10-CM diagnosis codes, opioid experience and options and discontinuation of therapy. At the end of each month the information from patients and the physician practice are combined to generate a set of standard practice reports detailing baseline characteristics of new patients and response to therapy for continuing patients disaggregated by target patient groups.

The registry can be interrogated for any patient who has enrolled in real time. Practices can generate reports on individual patients as well as on target patient groups. Handbooks are provided to the practice on the implementation and management of the registry as well as a comprehensive data dictionary. The registry and all data transfers both meet federal standards for privacy and the protection of health information. 


\section{The ASIPP Recommendations}

The 2017 ASIPP recommendations are designed to 'promote guidance for the prescription of opioids for the management of chronic non-cancer pain'. The guidance covers how to appropriately prescribe opioids together with recommendations for providing a systematic and standardized approach to establish an evidence base to support initial and continuing claims by the treating physician that the prescription of opioid formulations are medically necessary. At the same time the ASIPP recommendations are intended to support improvement in treatment together with a reduction in drug abuse and diversion.

Importantly, from a third party perspective (e.g., a state medical board), the guidelines are designed both to encourage and set standards for evidence in the management of chronic non-cancer pain. These standards apply both to the management of chronic pain in primary care or community medical practice as well as to specialist pain practices.

The ASIPP evidence recommendations cover:

- Initial steps in opioid therapy

- Assessment of effectiveness of long term opioid therapy

- Monitoring for adherence and side effects

- Final phase

\section{Establishing Medical Necessity: Initial Steps in Opioid} Therapy

The ASIPP guidelines provide an exhaustive list of activities recommended for the initial steps in the establishment of medical necessity in the prescribing of opioid therapy. The assessment for opioid therapy should include: (i) comprehensive medical history assessment and documentation; (ii) assessment of pain level and functional status by pain location; (iii) screening for the likelihood of opioid abuse risk; and (vi) initiation of prescription drug monitoring.

The ASIPP guidelines emphasize the importance from a practice perspective of not only meeting compliance standards but also establishing the medical necessity for opioid therapy. The CPMR provides the treating physician with a range of data points that can be evaluated as part of the assessment of medical necessity. It is not, however, the role of the CPMR to establish a case for medical necessity but merely to provide in a robust and structured format those data elements that may be judged pertinent in the decision. This allows not only consistency in reporting across target patient populations but an audit trail that can be activated to demonstrate that the requisite data elements are part of the medical record. Concordance between these recommendations and the patient and physician office data elements and reports that are inputs to the CPMR are detailed in Table 1.

\section{Satisfaction with Care and Treatment Goals}

The importance of establishing medical necessity in the initial decision to prescribe opioids is only the first step. The role of the CPMR as summarized in Table 2 is also to assess (i) the patient's satisfaction with care received and (ii) the extent to which a pain management plan has been agreed between provider and patient. Following each visit to the practice, the CPMR, over the course of treatment the patient completes an on line satisfaction questionnaire. This asks the patient to assess the quality of care from the provider and staff as well as issues of access to care and the communication between the provider and the patient. After the initial visit the questionnaire also includes a series of questions relating to the provision of a pain management plan, issues raised in the preparation of a plan and treatment goals.

\section{Assessment of Effectiveness of Long Term Opioid Therapy}

One of the major constraints on introducing patients to opioid therapy is the limited evidence for the risks and benefits of opioids in impacting both pain intensity and functional status in the medium to long-term; a situation which is even more constraining when questions are raised as to the risks and benefits of opioid use by specific pain locations. A further puzzling (and annoying) feature of the continued push for opioids as medically necessary is that this continued advocacy of opioids (both in the US and globally) occurs in an information vacuum when questions are raised as to the competing risks and benefits of opioid formulations (e.g., abuse deterrent technologies) as well as opioid versus nonopioid interventions. This point, as noted above, is emphasized both in the ASIPP guidelines but also in the recent CDC report on opioid guidelines. It is this dearth of information outside of short-term (and limited) clinical trials that the CPMR is intended to address. Even so, as the ASIPP guidelines note, this lack of randomized trials or even observational studies does not preclude the possible effectiveness of opioids in the medium and long-term treatment of chronic pain. The question is not just one of establishing initial medical necessity but of continued medical necessity over the course of treatment. Once introduced to an opioid, patients need to be closely monitored to assess the impact, as detailed in the ASIPP guidelines on: (i) analgesia; (ii) activity; (iii) aberrant behavior; and (iv) adverse effects. The CPMR captures all of these data points over the course of treatment (Table 3).

\section{Monitoring for Adherence and Side Effects}

On the evidence available, the ASIPP recommendations caution against the prescribing of long-acting and high-dose opioids. As detailed by ASIPP, available evidence points to a similar effectiveness between short-acting and longacting opioids with an increased prevalence of adverse outcomes with long-acting, high-dose formulations. The recommendation is that long-acting high-dose opioids should only be used with severe intractable pain that is not responsive to short-acting opioids or moderate dosing of long-acting formulations. Unless, therefore, a case for the initial 
prescribing of high-dose long acting opioid formulations can be made on the grounds of medical necessity, the ASIPP argument is one for continued feedback to the treating physician where the initial decision may be for a short-acting opioid at a low or medium dose with a possible transition to higher doses or a medium dose long-acting formulation. The CPMR provides this feedback in tracking dose formulations over the course of treatment, matching these to the response reported by the patient. Although not covered in the ASIPP guidelines, the CPMR supplements the pain level and functional status measures reported by pain location with the Patients' Global Impression of Change (PGIC) scale ${ }^{7}$.

As well as tracking patient response in terms of intensity of pain and functional status, overall response to therapy (PGIC) and aberrant medical behavior, the CPMR also tracks therapy modifications, adverse events and referrals. The CPMR, in tracking patients over the course of therapy will also capture adherence to the prescribed opioid dosing and discontinuation of therapy.

\section{Final Phase in Treatment}

The practice is asked to report on patients whose treatment has terminated or who have not scheduled a follow up visit. This is important because little is known as to why patients 'drop off the radar screen' after being initiated to and treated with opioids as well as other chronic pain interventions. If a follow-up visit has not been scheduled then reasons for not rescheduling are presented in the CPMR. These range from satisfactory/unsatisfactory resolution of pain and functional status symptoms, adverse events, refusal to consider alternative treatments and the patient moving to another practice. Capturing this 'final phase' in opioid management is important when it comes to evaluating the overall effectiveness of opioid medications in treatment practice. Physicians and physician practices should not only be compliant in initiating patients to opioid therapy and monitoring their response to therapy, but also compliant in 'closing the books'. This does not mean, of course, that the patient is put to one side, particularly if the reason for discontinuation is that symptoms have not been resolved. The advantage with the CPMR is that it is still possible to follow up patients to assess their ongoing pain and functional status, and how they are coping.

\section{Conclusions}

The CPMR has been developed as a necessary tool for the management of chronic pain in real-time. Its primary purpose is to evaluate response to therapy given initial presentation of pain and functional status by pain location and functional status. Achieving this will go a long way to meeting the evidence gap for outcomes in the medium to long-term for chronic pain interventions. In the case of opioids, the CPMR provides an evidence platform that allows physician practices to demonstrate the initial case, or medical necessity, for prescribing opioids and, in tracking patients over time, the case for continued medical necessity in opioid prescribing.

The question of physician compliance is central to demonstrating medical necessity. Unless a physician or physician practice can demonstrate that it meets guidelines for the prescribing of opioids then there may be practice risks associated with the quality of care. There is the potential liability for inappropriate opioid prescribing but also risks that the patients may not be appropriately monitored and managed over the course of their treatment. Continuing medical necessity in the prescribing of opioids, as the ASIPP guidelines point out involves being able to demonstrate from the patient record that 'appropriate analgesic and functional status is achieved either with opioid therapy alone or in conjunction with other modalities'. The ability to quantify, with validated instruments and reporting, that a minimum improvement is being achieved is critical for continued opioid therapy. This has, of course, to be put against risks of medication abuse and unacceptable side effects. Continuous monitoring and reporting from both the patient and from the practice as inputs to the CPMR achieve not only support claims for medical necessity but also demonstrate the commitment of the physician and physician practice to meeting compliance standards.

Conflicts of Interest: None reported. 
Table 1

Chronic Pain Management Registry: Concordance with ASIPP Recommendations for Initial Steps to Establish Medical Necessity

\begin{tabular}{|c|c|}
\hline \multicolumn{2}{|c|}{ ASIPP Recommendations: Comprehensive Patient Assessment } \\
\hline General Medical History & $\begin{array}{l}\text { Patients report on their overall current health status (Excellent, Very Good, Good, Fair, } \\
\text { Poor) and how this compares to } 12 \text { months ago (Better, Worse, About the same, Don't } \\
\text { Know). Prior opioid use is requested together with opioid use in the past } 30 \text { days. } \\
\text { Physician practices can request additional medical history data. }\end{array}$ \\
\hline Informed Consent & $\begin{array}{l}\text { The CPMR provides forms to be completed for on-line signature. These include patient } \\
\text { consent (including nominated pharmacy), opioid agreement (see below), practice } \\
\text { financial policy, privacy notice(s) for protected health information, prescription and OTC } \\
\text { medications and consent for surgical interventions. }\end{array}$ \\
\hline $\begin{array}{l}\text { Psychosocial History and } \\
\text { Psychological Evaluation }\end{array}$ & $\begin{array}{l}\text { Psychosocial experience captured as part of risk assessment: previous discharge from } \\
\text { medical practice; medication behavior; depression/anxiety; drinking or drug abuse } \\
\text { problems (including parents); jail or prison time; reading ability. Patients are asked to } \\
\text { complete validated PROs for: (i) fatigue; (ii) anxiety; and (iii) depression } 89 \text { 10. Patients } \\
\text { are evaluated for the views on pain medications utilizing a validated PRO instrument }{ }^{11}\end{array}$ \\
\hline Previous pain interventions & $\begin{array}{l}\text { The patient is asked to report on previous interventions to treat their current pain to } \\
\text { include prescription medication, over-the-counter medication use, procedures, } \\
\text { acupuncture, physical therapy, exercise and psychological counseling. }\end{array}$ \\
\hline Pain Condition & $\begin{array}{l}\text { The CPMR asks patients to report on the overall level of pain to include: (i) present pain } \\
\text { experience and; (ii) chronic pain experience and (iii) likelihood of the presence of } \\
\text { neuropathic/fibromyalgic pain }{ }^{12} \text {. Responses are summarized and scored and submitted } \\
\text { to treating physician prior to initial visit. Score: current pain intensity, Chronic Graded } \\
\text { Pain Scale and likelihood of continuing chronic pain }{ }^{13} 14 \text {. Patients are asked to describe } \\
\text { the location of their pain, pain level and intensity by location and to assess functional } \\
\text { (see below). Multiple pain locations can be reported on. Responses are summarized and } \\
\text { scored utilizing PRO algorithms and reported prior to the initial visit for that location. }\end{array}$ \\
\hline Functional Status & $\begin{array}{l}\text { Pain level and functional status can be reported for up to } 13 \text { body pain locations. } \\
\text { Functional status is reported prior to each practice visit utilizing validated PROs specific } \\
\text { to that pain location. Responses are summarized and scored for the treating physician. }\end{array}$ \\
\hline Sleep Patterns & Sleep experience is reported and summarized utilizing a validated PRO ${ }^{15}$ \\
\hline Constipation & A validated PRO assess constipation status at each visit ${ }^{16}$ \\
\hline \multicolumn{2}{|c|}{ ASIPP Recommendations: Opioid Risk Abuse Screening } \\
\hline $\begin{array}{l}\text { Risk Assessment for Aberrant opioid } \\
\text { Behavior }\end{array}$ & $\begin{array}{l}\text { Patients complete a validated risk assessment instrument prior to their initial visit }{ }^{17} \text {. } \\
\text { Assessed as: low risk, medium risk or high risk for abuse }\end{array}$ \\
\hline Substance Use History & $\begin{array}{l}\text { Patients are asked, as part of the risk assessment, if they have ever had a drinking or drug } \\
\text { abuse problem, whether their biological parents have had a drinking or drug abuse } \\
\text { problem and whether they have spent time in jail or prison. }\end{array}$ \\
\hline Opioid Utilization & $\begin{array}{l}\text { Patients are asked prior to their initial visit if they have ever been prescribed opioids } \\
\text { (from a list of } 12 \text { formulations with brand names) and used any of these opioid } \\
\text { formulations in the past } 30 \text { days. On subsequent visits asked what their pain medication } \\
\text { behavior has involved in the last } 30 \text { days or since their last visit (to identify aberrant } \\
\text { behavior) }\end{array}$ \\
\hline Opioid Agreement & $\begin{array}{l}\text { Patients are provided with an opioid use contract and the practice is asked if a contract } \\
\text { has been completed }\end{array}$ \\
\hline Prescription Monitoring Program & $\begin{array}{l}\text { Practices are asked to report if a check has been made through the state prescription } \\
\text { drug monitoring program }\end{array}$ \\
\hline Urine Drug Screening & Practices are asked to report if a urine test has been taken \\
\hline
\end{tabular}




\section{Table 2}

Chronic Pain Management Registry: Concordance with ASIPP Recommendations for Establishing Treatment Goals

\begin{tabular}{|l|l|}
\hline ASIPP Recommendations: Pain Management and Treatment Goals \\
\hline Pain Management Plan & $\begin{array}{l}\text { Following the initial encounter with the treating physician, the patient is asked to } \\
\text { complete on-line a brief questionnaire covering satisfaction with care and whether or not } \\
\text { a pain management plan was discussed with the provider and agreed with the patient. } \\
\text { The pain management questions (16 in total) not only ask whether a pain management } \\
\text { plan was agreed but also questions on the extent to which treatment options were } \\
\text { reviewed, the benefits and risks of alternative treatments (e.g., abuse deterrent opioid } \\
\text { formulations), the anticipated length of treatment, the impact of comorbid disease states } \\
\text { and possible involvement of other health care professionals. }\end{array}$ \\
\hline Measurement and Treatment & $\begin{array}{l}\text { The CPMR provides the framework for not only establishing treatment goals in terms of } \\
\text { potential target reductions in pain intensity and functional status by pain location, but } \\
\text { also provides benchmarks for minimal clinical improvement for the respective validated } \\
\text { scales (the ASIPP guidelines propose as a rule of thumb a 30\% improvement in a pain } \\
\text { score as the target minimum improvement). The CPMR (as detailed below) provides } \\
\text { these details at each follow up visit by comparing current pain intensity and functional } \\
\text { status scores to baseline in the pre-visit report to the practice. }\end{array}$ \\
\hline Return to work & $\begin{array}{l}\text { Although not considered explicitly by the ASIPP guidelines, the CPMR asks respondents } \\
\text { their employment status at each visit so that if return to work is a goal this can be } \\
\text { tracked over the course of therapy. }\end{array}$ \\
\hline
\end{tabular}

Table 3

Chronic Pain Management Registry: Concordance with ASIPP Recommendations for Assessing the Continued Effectiveness of Opioid Therapy

\begin{tabular}{|c|c|}
\hline \multicolumn{2}{|c|}{ ASIPP Recommendations: Assessing Opioid Continued Medical Necessity } \\
\hline Analgesia: Pain Level & $\begin{array}{l}\text { Prior to each practice visit the patient reports on pain intensity by pain location carrying } \\
\text { forward the initial pain intensity status and comparing that to the pre-visit status for all } \\
\text { follow-up visits. }\end{array}$ \\
\hline Activity: Functional Status & $\begin{array}{l}\text { Prior to each practice visit the patient reports on functional status by pain location carrying } \\
\text { forward the initial functional status score and comparing that to the pre-visit status score for } \\
\text { all follow up visits. }\end{array}$ \\
\hline Aberrant Medication Behavior & $\begin{array}{l}\text { Prior to each follow up visit over the course of treatment, patients report on their medication } \\
\text { aberrant behavior; this reported to the treating physician prior to the practice encounter }\end{array}$ \\
\hline Adverse Effects & $\begin{array}{l}\text { Dose related adverse events are recorded by the physician/practice following each encounter } \\
\text { with the patient and coded to ICD-10-CM standards. }\end{array}$ \\
\hline $\begin{array}{l}\text { Overall Response to Therapy: } \\
\text { PGIC }\end{array}$ & $\begin{array}{l}\text { Prior to each visit the patient completes the PGIC scale. This asks patients to indicate, since } \\
\text { beginning their treatment how they would describe the change (if any) in activity limitations, } \\
\text { symptoms, emotions and overall quality of life related to their pain condition. A seven point } \\
\text { response scale ranges from "No change (or condition got worse)' to 'A great deal better and a } \\
\text { considerable improvement that has made all the difference'. }\end{array}$ \\
\hline \multicolumn{2}{|c|}{ ASIPP Recommendations: Physician/Practice Compliance and Physician/Practice Response } \\
\hline $\begin{array}{l}\text { Analgesia: Pain Level and } \\
\text { Functional Status }\end{array}$ & $\begin{array}{l}\text { After each visit the physician/practice reports on medication utilization to identify opioid } \\
\text { formulation, dosing, number of tablets/polls per day, days supplied, whether titration is } \\
\text { occurring and if the medication is for rescue purposes. This allows the change in pain level to } \\
\text { be matched to dosing regimen and the medical necessity of continued opioid use considered. }\end{array}$ \\
\hline Titration & $\begin{array}{l}\text { The CPMR asks the physician practice to report titration and allow this to be tracked for the } \\
\text { patient over the course of treatment. }\end{array}$ \\
\hline
\end{tabular}




\begin{tabular}{|l|l|}
\hline $\begin{array}{l}\text { Tapering and Discontinuation } \\
\text { of Opioid Therapy }\end{array}$ & $\begin{array}{l}\text { After each visit the /physician practice is asked to report on any activities directed towards } \\
\text { tapering or discontinuation of opioid therapy. The practice is given a number of options to } \\
\text { report including failure to achieve significant analgesia/functioning, excessive dosing to } \\
\text { achieve targets, presence of risk factors (e.g., sleep apnea), medication aberrant behavior and } \\
\text { failure to meet opioid agreement standards. }\end{array}$ \\
\hline Therapy options to opioids & $\begin{array}{l}\text { After each visit the practice is asked to report on whether at that visit the patient was offered } \\
\text { alternative non-opioid interventions in order to resolve issues with pain experience and/or } \\
\text { functionality. If alternatives were offered then they are to be detailed. }\end{array}$ \\
\hline $\begin{array}{l}\text { Scheduling and Treatment } \\
\text { Termination }\end{array}$ & $\begin{array}{l}\text { At each visit the physician is asked to report whether or not a further visit has been scheduled } \\
\text { for the patient. }\end{array}$ \\
\hline ASIPP Recommendations: Long Acting and High Dose Opioids \\
\hline $\begin{array}{l}\text { Tracking formulations and } \\
\text { dosing }\end{array}$ & $\begin{array}{l}\text { At each visit the practice reports on the medications current prescribed to include opioid } \\
\text { formulation, dosing and number of tablets/pills prescribed. The CPMR allows the physician to } \\
\text { track dosing over the course of therapy. The platform can be customized if a practice wishes } \\
\text { to flag low dose (e.g. <40 MME), moderate dose (41 to } 90 \text { MME) or high dose (>90 MME) } \\
\text { patients. }\end{array}$ \\
\hline Therapy modifications & $\begin{array}{l}\text { The physician practice is required to report on all modifications to the initial therapy regimen, } \\
\text { to detail and medication supplementation and the report on whether or not alternative } \\
\text { treatment interventions were offered and accepted. }\end{array}$ \\
\hline
\end{tabular}

\section{References}

${ }^{1}$ Medical Board of California. Guidelines for prescribing controlled substances for pain. November 2014

2 Dowell D, Haegerich TM, Chou R. CDC Guideline for Prescribing Opioids for Chronic Pain - United States, 2016. MMWR Recomm Rep. 2016; ePub: March 2016: DOI: http://dx.doi.org/10.15585/mmwr.rr6501e1er

${ }^{3}$ Dijulio B, Wu B, Brodie M. The Washington Post/Kaiser Family Foundation survey of long-term prescription painkiller use and their household members. Kaiser Family Foundation, December 2016 http://files.kff.org/attachment/Survey-of-Long-Term-PrescriptionPainkiller-Users-and-Their-Household-Members

${ }^{4}$ Langley PC. A practice based chronic pain management registry (CPMR): Structure and content of a proposed platform Inov Pharm. $2019 ; 10(1)$ : Article 11

${ }^{5}$ Manchitkanti L, Kaye A, Knezevic N et al. Responsible, safe and effective prescription of opioids for chronic non-cancer pain: American Society of Interventional Pain Physicians (ASIPP) Guidelines. Pain Physician. 2017;20:S3-S92

${ }^{6}$ Hurst $\mathrm{H}$, Bolton J. Assessing the clinical significance of change scores recorded on subjective outcome measures. $J$ Manipoulative Physiol Ther. 2004;27:26-35

${ }^{7}$ Hurst $\mathrm{H}$, Bolton J. Assessing the clinical significance of change scores recorded on subjective outcome measures. J Manipulative Physiol Ther. 2004;27:26-35

${ }^{8}$ Schwartz JE, Jandorf L, Krupp LB. The measurement of fatigue: a new instrument. J Psychosom Res. 1993;37:753-62

${ }^{9}$ Kroenke K, Spitzer R, Williams JB. The PHQ-9: validity of a brief depression severity measure. J Gen Int Med. 2001;16:606-16

${ }^{10}$ Kroenke K, Spitzer R, Williams J et al. A brief measure for assessing generalized anxiety. Arch Int Med. 2006;166:1092-7

${ }^{11}$ Elander J, Said O, Maratos F, Dys A et al. Development and validation of a short-form Pain Medication Attitudes Questionnaire (PMAQ-14). Pain. 2017;158(3):400-407 
12 Portenoy R. Development and testing of a neuropathic pain screening questionnaire: ID Pain. Curr Med Res Opin. 2006;22(8):155565

${ }^{13}$ Von Korff M, Ormel J, Keefe F et al. Grading the severity of chronic pain. Pain. 1992;50:133-49

${ }^{14}$ Von Korff M, Dunn K. Chronic pain reconsidered. Pain. 2008;138(2):267-76

${ }^{15}$ Hays RD, Stewart AL. Sleep measures in A L Stewart and J E Ware (eds). Measuring functioning and well-being: The Medical Outcomes Study Approach (pp. 235-59). Durham, NC: Duke University Press, 1992

${ }^{16}$ Frank L, Kleinman L, Farup C et al. Psychometric validation of a constipation symptom assessment questionnaire. Scand J Gastroenterol. 1999;34:870-7

17 Jones T, Schmidt M, Moore T. Further validation of an opioid risk assessment tool: The Brief Risk Questionnaire. Ann Psychiatryt Ment Health. 2015;3(3):1032 